\title{
A MAYER-VIETORIS SEQUENCE IN GROUP HOMOLOGY AND THE DECOMPOSITION OF RELATION MODULES
}

\author{
by A. J. DUNCAN, ${ }^{*}$ GRAHAM J. ELLIS $\dagger$ and N. D. GILBERT
}

(Received 17 September, 1993; revised 4 July, 1994)

Introduction. W. A. Bogley and M. A. Gutierrez [2] have recently obtained an eight-term exact homology sequence that relates the integral homology of a quotient group $\Gamma / M N$, where $M$ and $N$ are normal subgroups of the group $\Gamma$, to the integral homology of the free product $\Gamma / M * \Gamma / N$ in dimensions $\leq 3$ by means of connecting terms constructed from commutator subgroups of $\Gamma, M, N$ and $M \cap N$. In this paper we use the methods of [4] to recover this exact sequence under weaker hypotheses and for coefficients in $\mathbb{Z} / q \mathbb{Z}$ for any non-negative integer $q$. Further, for $q=0$ we extend the sequence by three terms in order to capture the relation between the fourth homology groups.

Our principal application of the Mayer-Vietoris sequence is to relation modules. If $1 \rightarrow R \rightarrow F \rightarrow G \rightarrow 1$ is a free presentation of a group $G$, then the relation module is $R^{a b}$ endowed with the $G$-action obtained from the conjugation action of the free group $F$ on the normal subgroup $R$. There exists an extensive theory of relation modules for finite groups $G$ (see [10]) and interesting results for classes of infinite groups, such as for polycyclic groups in [17]. A central theme of the theory has been the study of indecomposable relation modules. In the present paper we address the complementary issue: we give conditions that guarantee the decomposition of a relation module as a direct sum of submodules. In particular, as a simple consequence of the Mayer-Vietoris sequence, we see that the relation module of a presentation expressed as the union of two subpresentations is a direct sum of submodules induced from the relation modules of the subpresentations, provided that the relation subgroups of the two subpresentations are independent in the ambient free group.

In Section 2 we discuss independence of normal subgroups of free groups in more detail, and prove the equivalence between a relation module decomposition as a direct sum of submodules induced from the relation modules of subpresentations and independence of the relation subgroups. Whilst our methods here are essentially elementary, the main theorem applies to interesting classes of presentations, and some applications are explored in Section 3. In favourable cases the connecting terms can be precisely described, and are often trivial, indicating that independence of relation subgroups is reflected in higher-order independence conditions.

For a survey of matters closely related to the theme of this paper, the reader is referred to [3]. The authors are grateful to W. A. Bogley and to J. Howie for conversations and helpful comments.

\footnotetext{
* Research partially supported by a U.K. Science and Engineering Research Council Postdoctoral Fellowship at Heriot-Watt University.

† The second author thanks the Mathematics Department of Northwestern University, where research for this paper was carried out, for their hospitality. Additional support from the Centenary Fund of the Edinburgh Mathematical Society is gratefully acknowledged.
}

Glasgow Math. J. 37 (1995) 159-171. 
1. A Mayer-Vietoris sequence in group homology. Let $q$ be a fixed non-negative integer. For any group $\Gamma$ we denote by $H_{n}\left(\Gamma, \mathbb{Z}_{q}\right)$ the homology of $\Gamma$ with coefficients in the trivial $\Gamma$-module $\mathbb{Z}_{q}=\mathbb{Z} / q \mathbb{Z}$. If $N$ is a normal subgroup of $\Gamma$ then $\Gamma \#_{q} N$ denotes the subgroup of $\Gamma$ generated by the commutators $[g, n]$ and the elements $n^{q}$, where $g \in \Gamma$ and $n \in N$. Note that $\Gamma \#_{q} N$ is normal in $\Gamma$, and that when $q=0$ we have $\Gamma \#_{q} N=[\Gamma, N]$.

THEOREM 1.1. Let $\Gamma$ be a group and let $M$ and $N$ be normal subgroups of $\Gamma$. Then there is a natural exact sequence of groups:

$$
\begin{aligned}
& H_{4}\left(\Gamma / M, \mathbb{Z}_{q}\right) \oplus H_{4}\left(\Gamma / N, \mathbb{Z}_{q}\right) \rightarrow H_{4}\left(\Gamma / M N, \mathbb{Z}_{q}\right) \rightarrow V_{3} \rightarrow H_{3}\left(\Gamma / M, \mathbb{Z}_{q}\right) \oplus H_{3}\left(\Gamma / N, \mathbb{Z}_{q}\right) \\
& \quad \rightarrow H_{3}\left(\Gamma / M N, \mathbb{Z}_{q}\right) \rightarrow V_{2} \rightarrow H_{2}\left(\Gamma / M, \mathbb{Z}_{q}\right) \oplus H_{2}\left(\Gamma / N, \mathbb{Z}_{q}\right) \rightarrow H_{2}\left(\Gamma / M N, \mathbb{Z}_{q}\right) \\
& \rightarrow V_{1} \rightarrow H_{1}\left(\Gamma / M, \mathbb{Z}_{q}\right) \oplus H_{1}\left(\Gamma / N, \mathbb{Z}_{q}\right) \rightarrow H_{1}\left(\Gamma / M N, \mathbb{Z}_{q}\right) \rightarrow 0
\end{aligned}
$$

such that

(i) there is an isomorphism $V_{1} \rightarrow \Gamma /(M \cap N)\left(\Gamma \#_{q} \Gamma\right)$,

(ii) there is a surjection $V_{2} \rightarrow\left(M \cap N \cap\left(\Gamma \#_{q} \Gamma\right)\right) /[M, N]\left(\Gamma \#_{q}(M \cap N)\right)$ which is an isomorphism if $H_{2}\left(\Gamma, \mathbb{Z}_{q}\right)=0$,

(iii) for $q=0$ there is a surjection $V_{3} \rightarrow \operatorname{ker}(\bigwedge(\Gamma ; M, N) \rightarrow \bigwedge(\Gamma ; \Gamma, \Gamma))$ which is an isomorphism if $H_{3}(\Gamma, \mathbb{Z})=0$.

Parts (i) and (ii) of Theorem 1.1 for $q=0$ are a slight generalisation of Theorem 5.2 of [2]. Part (iii), and parts (i) and (ii) for $q \neq 0$, are new.

In the following discussion, up to and including Corollary 1.2, we fix $q=0$, and write $H_{n}(\Gamma)$ for the homology of a group $\Gamma$ with integral coefficients.

The group $\wedge(\Gamma ; M, N)$ in part (iii) of the theorem has a presentation with generators $x \wedge y$, where $(x, y) \in(M \times N) \cup(N \times M) \cup(\Gamma \times M \cap N) \cup(M \cap N \times \Gamma)$, subject to the relations

$$
\begin{aligned}
x x^{\prime} \wedge y & =\left(x x^{\prime} x^{-1} \wedge x y x^{-1}\right)(x \wedge y), \\
x \wedge y y^{\prime} & =(x \wedge y)\left(y x y^{-1} \wedge y y^{\prime} y^{-1}\right), \\
x \wedge y & =(y \wedge x)^{-1}, \\
(x \wedge y)(u \wedge v)(y \wedge x) & =[x, y] u[y, x] \wedge[x, y] v[y, x], \\
z \wedge z & =1, \text { if } z \in M \cap N .
\end{aligned}
$$

The homomorphism $\bigwedge(\Gamma ; M, N) \rightarrow \bigwedge(\Gamma ; \Gamma, \Gamma)$ in (iii) is the canonical one. The group $\bigwedge(\Gamma ; \Gamma, \Gamma)$ is isomorphic to the group $(\Gamma, \Gamma)$ introduced by $C$. Miller [18] to give an interpretation of $H_{2}(\Gamma)$, and to the group $\Gamma \wedge \Gamma$ of $[5]$. As in $[5,18]$, we have

$$
H_{2}(\Gamma) \cong \operatorname{ker}(\bigwedge(\Gamma ; \Gamma, \Gamma) \rightarrow \Gamma) .
$$

Hence if $H_{2}(\Gamma)=0=H_{3}(\Gamma)$ then $V_{3} \cong \operatorname{ker}(\bigwedge(\Gamma ; M, N) \rightarrow \Gamma)$.

If $M \cap N=1$ then $\bigwedge(\Gamma ; M, N)$ is isomorphic to the tensor product $M^{a b} \otimes N^{a b}$ of 
abelian groups. If further $H_{2}(\Gamma)=0=H_{3}(\Gamma)$ then we obtain from Theorem 1.1 the two exact sequences:

$$
\begin{aligned}
& H_{4}(\Gamma / M) \oplus H_{4}(\Gamma / N) \rightarrow H_{4}(\Gamma / M N) \rightarrow M^{a b} \otimes N^{a b} \rightarrow H_{3}(\Gamma / M) \oplus H_{3}(\Gamma / N) \rightarrow H_{3}(\Gamma / M N) \rightarrow 0, \\
& 0 \rightarrow H_{2}(\Gamma / M) \oplus H_{2}(\Gamma / N) \rightarrow H_{2}(\Gamma / M N) \rightarrow H_{1}(\Gamma) \rightarrow H_{1}(\Gamma / M) \oplus H_{1}(\Gamma / N) \rightarrow H_{1}(\Gamma / M N) \rightarrow 0 .
\end{aligned}
$$

Following [11], we say that two normal subgroups $M, N$ of a group $\Gamma$ are independent if $M \cap N=[M, N]$. This notion is the main concern of Section 2. If $M$ and $N$ are independent, and if $H_{2}(\Gamma)=0=H_{3}(\Gamma)$, then $V_{2}=0$ and $V_{1}=H_{1}(\Gamma)$. If further $\Gamma=M N$ then we deduce from the Mayer-Vietoris sequence that

$$
\begin{gathered}
H_{3}(\Gamma / M) \oplus H_{3}(\Gamma / N) \cong \operatorname{ker}(\bigwedge(\Gamma ; M, N) \rightarrow \Gamma), \\
H_{2}(\Gamma / M)=0=H_{2}(\Gamma / N), \\
H_{1}(\Gamma) \cong H_{1}(\Gamma / M) \oplus H_{1}(\Gamma / N) .
\end{gathered}
$$

These observations pertain to relation modules. Given free presentations

$$
1 \rightarrow R_{i} \rightarrow F \rightarrow G_{i} \rightarrow 1
$$

for $i=1,2$, with $R_{1}$ and $R_{2}$ independent, we take $\Gamma=R_{1} R_{2}$ and note that

$$
H_{1}\left(R_{1} R_{2} / R_{2}\right) \cong H_{1}\left(R_{1} /\left[R_{1}, R_{2}\right]\right) \cong R_{1}^{a b} \otimes_{R_{2}} \mathbb{Z} \cong R_{1}^{a b} \otimes_{G_{1}} \mathbb{Z} G
$$

where $G=F / \Gamma$. We deduce the following result.

COROLLARY 1.2. If $R_{1}$ and $R_{2}$ are independent normal subgroups of a free group $F$, with $G_{i}=F / R_{i}$ and $G=F / R_{1} R_{2}$, then

$$
\left(R_{1} R_{2}\right)^{a b} \cong\left(R_{1}^{a b} \otimes_{G_{1}} \mathbb{Z} G\right) \oplus\left(R_{2}^{a b} \otimes_{G_{2}} \mathbb{Z} G\right) .
$$

In Section 2 we shall generalise this corollary to an independent family of normal subgroups of $F$, and establish the converse.

Proof of Theorem 1.1. Let $B(\Gamma)$ denote the classifying space of a group $\Gamma$. The exact sequence given in the theorem is the low-dimensional part of the exact homology sequence of the cofibration sequence

$$
B(\Gamma / M) \vee B(\Gamma / N) \rightarrow B(\Gamma / M N) \rightarrow D
$$

and to prove the theorem we need to identify $H_{i} D$ for $i=2,3,4$. We shall exhibit a space $C$ and a cofibration sequence

$$
D \rightarrow C \rightarrow \Sigma^{2} B(\Gamma)
$$

(where $\Sigma^{2}$ denotes the double suspension) and compute $H_{i}(C)$ for $i=2,3,4$. The 
statements of the theorem then follow from the exact homology sequence of the cofibration sequence (3). We shall first prove the theorem for $q=0$.

Suppose that $q=0$. Let $B(\Gamma ; M)$ denote, as in [4], the mapping cone of $B(\Gamma) \rightarrow$ $B(\Gamma / M)$. The space $C$ is the space $B(\Gamma ; M, N)$ of [4], constructed as the mapping cone of $B(\Gamma ; M) \rightarrow B(\Gamma / N ; M N / N)$ (or as the mapping cone of $B(\Gamma ; N) \rightarrow B(\Gamma / M, M N / M)$, which gives the same result). It follows that $C$ is 2-connected (as it is a mapping cone of 1-connected spaces), so that $\mathrm{H}_{2} \mathrm{C}=0$, and that $C$ fits into a commutative diagram in which every row and column is a cofibration sequence:

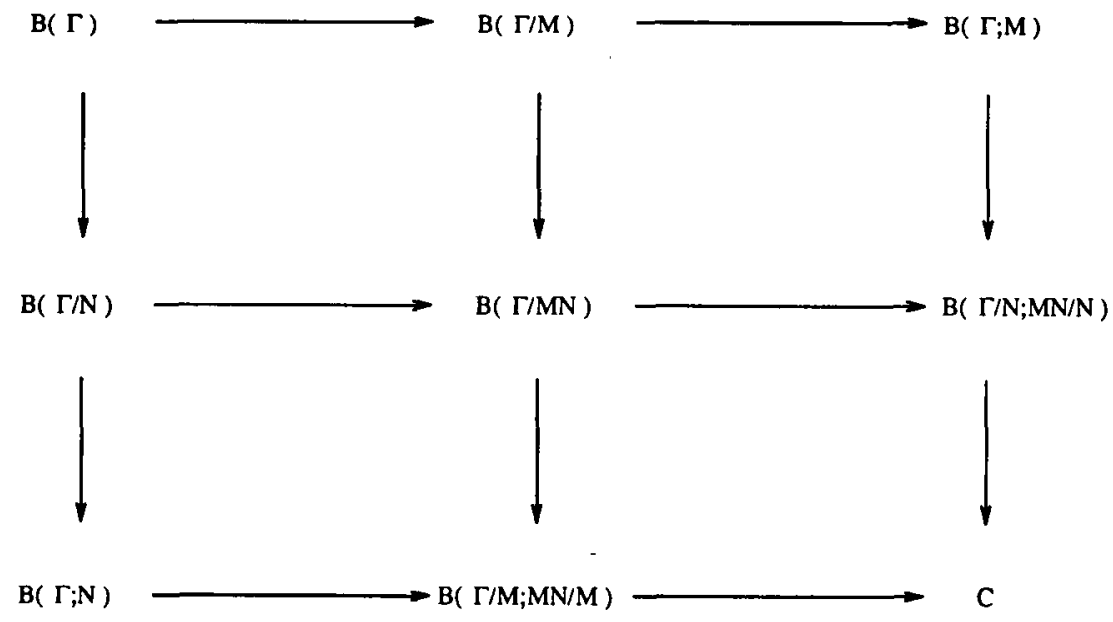

Figure 1.1

Now $D$ in (2) is the mapping cone of $B(\Gamma / N) \rightarrow B(\Gamma / M ; M N / M)$, and so there exists a map of cofibration sequences

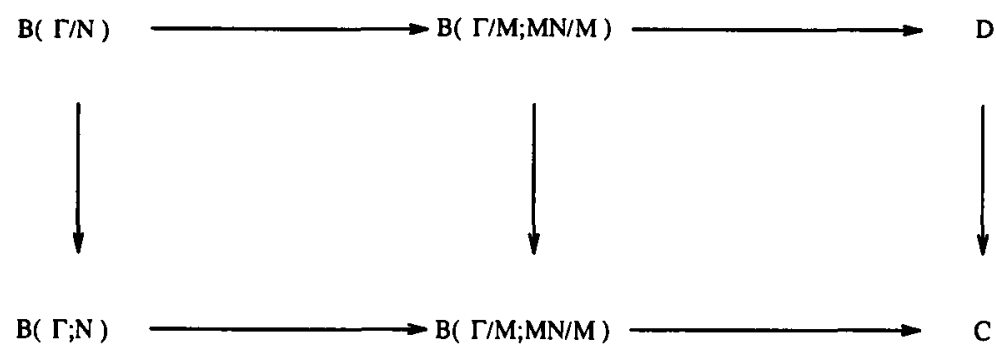

Figure 1.2

and the mapping cones of the vertical maps form the cofibration sequence

$$
\Sigma B(\Gamma) \rightarrow * \rightarrow \Sigma^{2} B(\Gamma)
$$

from which (3) follows. 
We compute $H_{i} C$ from $\pi_{i} C$. Consider the cubical diagram of spaces

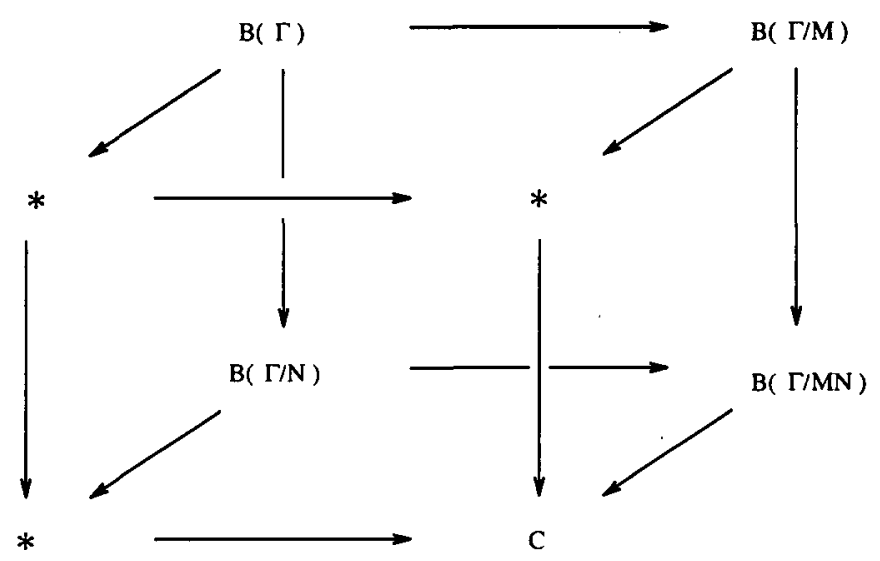

Figure 1.3

We enlarge this diagram by taking homotopy fibres:

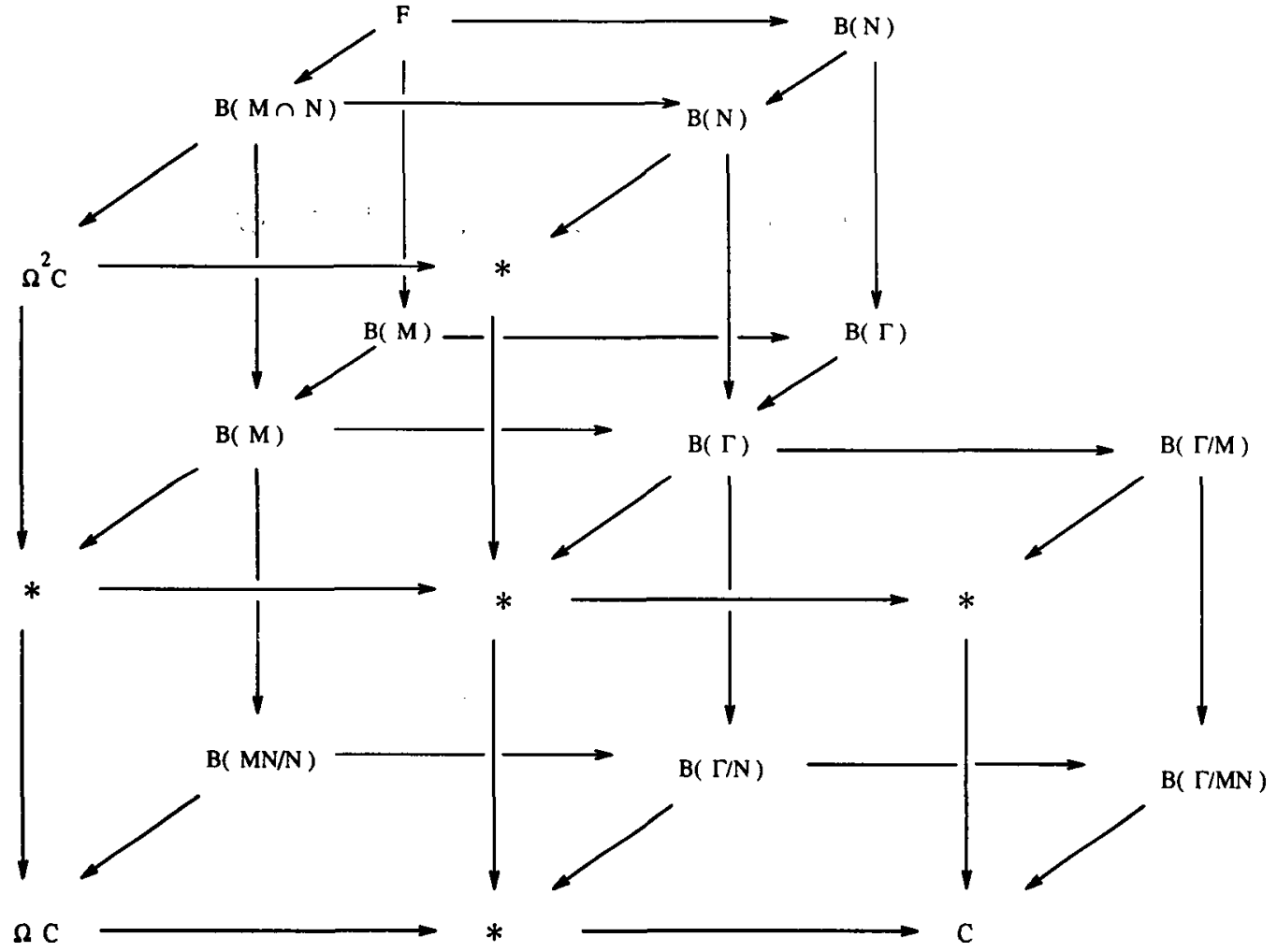

Figure 1.4

and extract the fibration sequence

$$
F \rightarrow B(M \cap N) \rightarrow \Omega^{2} C
$$


for further perusal. We see that $\pi_{i+3} C \cong \pi_{i} F$ for $i \geq 2$, and the exact homotopy sequence ends

$$
0 \rightarrow \pi_{4} C \rightarrow \pi_{1} F \rightarrow M \cap N \rightarrow \pi_{3} C \rightarrow 0 .
$$

A presentation of $\pi_{1} F$, and a description (in terms of this presentation) of the map $\pi_{1} F \stackrel{\not}{\rightarrow} M \cap N$ is given in Theorem 2.3 of [8]. From this description it is immediate that $\gamma$ has image $[M, N][\Gamma, M \cap N]$, whence

$$
H_{3} C \cong \pi_{3} C \cong M \cap N /[M, N][\Gamma, M \cap N]
$$

by the Hurewicz Theorem. Furthermore, $\pi_{4} C \cong \operatorname{ker} \gamma$. J. H. C. Whitehead's exact sequence [19] starts

$$
\ldots \rightarrow \pi_{3} C \otimes \mathbb{Z}_{2} \rightarrow \pi_{4} C \rightarrow H_{4} C \rightarrow 0
$$

and as in Lemma 4.2 of [5] the image of $\pi_{3} C \otimes \mathbb{Z}_{2}$ in $\pi_{4} C$ can be explicitly identified in $\pi_{1} F$. It follows that

$$
H_{4} C \cong \operatorname{ker}(\bigwedge(\Gamma ; M, N) \rightarrow \Gamma) .
$$

Now the map $\mathrm{H}_{4} \mathrm{C} \rightarrow \mathrm{H}_{2} \mathrm{G}$ in the exact homotopy sequence of 2 can be identified as the restriction of the canonical homomorphism $\bigwedge(\Gamma ; M, N) \stackrel{\alpha}{\rightarrow} \bigwedge(\Gamma ; \Gamma, \Gamma)$, since by [18] there is an isomorphism $H_{2} \Gamma \cong \operatorname{ker}(\bigwedge(\Gamma ; \Gamma, \Gamma) \rightarrow G)$. Now $\operatorname{ker} \alpha \leq \operatorname{ker}(\bigwedge(\Gamma ; M, N) \rightarrow \Gamma)$, and part (iii) of the theorem follows.

Suppose now that $q \geq 1$. The short exact sequence of coefficient modules

$$
0 \rightarrow \mathbb{Z} \stackrel{\times q}{\longrightarrow} \mathbb{Z} \rightarrow \mathbb{Z}_{q} \rightarrow 0
$$

induces a long exact sequence in the homology of the space $C$, part of which is

$$
\cdots \rightarrow H_{3}(C, \mathbb{Z}) \stackrel{\times q}{\longrightarrow} H_{3}(C, \mathbb{Z}) \rightarrow H_{3}\left(C, \mathbb{Z}_{q}\right) \rightarrow 0 .
$$

It follows that $H_{3}\left(C, \mathbb{Z}_{q}\right)=M \cap N /[M, N]\left(\Gamma \#_{q}(M \cap N)\right)$. There is also an induced long exact sequence in the homology of $D$, part of which is

$$
\ldots \rightarrow H_{2}(D, \mathbb{Z}) \stackrel{\times q}{\longrightarrow} H_{2}(D, \mathbb{Z}) \rightarrow H_{2}\left(D, \mathbb{Z}_{q}\right) \rightarrow 0 .
$$

It follows that $H_{2}\left(D, \mathbb{Z}_{q}\right)=\Gamma /(M \cap N)\left(\Gamma \#_{q} \Gamma\right)$. Parts (i) and (ii) of the theorem for $q \geq 1$ now follow from the cofibration sequence (2).

2. Independent normal subgroups of free groups. Let $F$ be a free group with basis $\mathbf{x}$ and suppose that $\left\{R_{1}, \ldots, R_{n}\right\}$ is a family of normal subgroups of $F$, with $n>1$. We write $R$ for the product $R_{1} \ldots R_{n}$ of all the $R_{i}$ with $1 \leq i \leq n$, and $N_{i}$ for the product $R_{1} \ldots R_{i-1} R_{i+1} \ldots R_{n}$ that omits $R_{i}$. Since each $R_{i}$ is normal, the order of the factors in such products is irrelevant. The quotient $F / R$ we denote by $G$, and the quotient $F / R_{i}$ by $G_{i}$. The family $\left\{R_{1}, \ldots, R_{n}\right\}$ is independent if, for all $i$ with $1 \leq i \leq n$, we have $R_{i} \cap N_{i}=\left[R_{i}, N_{i}\right]$. This notion has been studied in [1], [9], [11] and [16]. Independence may be considered as ensuring that certain intersections are as small as possible, or as ensuring that certain commutator subgroups are as large as possible: the following 
remarks will indicate that the second point of view is generally the most appropriate. None of these remarks depends upon the freeness of $F$.

\section{REMARKS}

1. The trivial group may be added to, or deleted from, an independent family to leave an independent family.

2. If $\left\{R_{1}, \ldots R_{n}\right\}$ is an independent family with $K$ a normal subgroup of $F$ contained in $R_{1}$, and if $\left\{K, R_{2}, \ldots, R_{n}\right\}$ is independent, then $K \cap\left[R_{1}, N_{1}\right]=\left[K, N_{1}\right]$.

We now state the generalisation of Corollary 1.2.

THEOREM 2.1. Let $F$ be a free group and $\left\{R_{1}, \ldots, R_{n}\right\}$ a family of normal subgroups of $F$, with $n>1$. Then the following are equivalent:

(1) the family $\left\{R_{1}, \ldots, R_{n}\right\}$ is independent,

(2) the quotient maps $R \rightarrow R / N_{i},(1 \leq i \leq n)$ induce an isomorphism

$$
R^{a b} \cong \bigoplus_{i=1}^{n} R_{i}^{a b} \otimes_{\mathbb{Z} G_{i}} \mathbb{Z} G
$$

of $\mathbb{Z} G$-modules.

Proof. (1) $\Rightarrow(2)$. The quotient maps $R \rightarrow R / N_{i}$ certainly induce a surjection $\theta: R^{a b} \rightarrow$ $\bigoplus_{i=1}^{n}\left(R / N_{i}\right)^{a b}$ and, as in (1) in Section 1 there is an isomorphism of $\mathbb{Z} G$-modules $\left(R / N_{i}\right)^{a b} \cong R_{i}^{a b} \otimes_{G_{i}} \mathbb{Z} G$. So it suffices to show that $\theta$ is an isomorphism of $\mathbb{Z} G$-modules. Since the $\mathbb{Z} G$-action on both $R^{a b}$ and $\left(R / N_{i}\right)^{a b}$ is induced by conjugation in $F$, it follows that $\theta$ is a $\mathbb{Z} G$-map. The kernel of $\theta$ is isomorphic to

$$
\frac{N_{1} \cap \ldots \cap N_{n}}{[R, R] \cap N_{1} \cap \ldots \cap N_{n}}
$$

so that it suffices to show that $N_{1} \cap \ldots \cap N_{n} \subseteq[R, R]$. we set

$$
N_{i_{1}, \ldots, i_{k}}=\prod_{j \neq i_{1}, \ldots, i_{k}} R_{j}
$$

Lemma 2.2. For any subset $\left\{i_{1}, \ldots, i_{k}\right\} \subseteq\{1, \ldots, n\}$ we have

$$
N_{i_{1}} \cap \ldots \cap N_{i_{k}}=\left(R_{i_{1}} \cap N_{i_{1}}\right) \ldots\left(R_{i_{k}} \cap N_{i_{k}}\right) N_{i_{1} \ldots, i_{k}} .
$$

Proof of Lemma. For ease of notation we shall assume that $i_{j}=j$ and we proceed by induction on $k$. The case $k=1$ is clear. For $k \geq 1$ we assume that

$$
N_{1} \cap \ldots \cap N_{k} \cap N_{k+1}=\left(R_{1} \cap N_{1}\right) \ldots\left(R_{k} \cap N_{k}\right) N_{1, \ldots, k} \cap N_{k+1} .
$$

Suppose that $x$ lies in the right-hand side. Then

$$
x=r_{1} r_{2} \ldots r_{k} r_{k+1} \ldots r_{n}=r_{1}^{\prime} r_{2}^{\prime} \ldots r_{k-1}^{\prime} r_{k}^{\prime} r_{k+2}^{\prime} \ldots r_{n}^{\prime},
$$

where $r_{j}, r_{j}^{\prime} \in R_{j}$ and if $j \leq k, r_{j} \in R_{j} \cap N_{j}$. Then

$$
r_{k+1}=\left(r_{1} \ldots r_{k}\right)^{-1} r_{1}^{\prime} \ldots r_{k}^{\prime} r_{k+2}^{\prime} \ldots r_{n}^{\prime}\left(r_{k+2} \ldots r_{n}\right)^{-1} \in N_{k+1}
$$

and so $x \in\left(R_{1} \cap N_{1}\right) \ldots\left(R_{k} \cap N_{k}\right)\left(R_{k+1} \cap N_{k+1}\right) N_{1, \ldots, k, k+1}$. This completes the inductive step and establishes one inclusion of the lemma, whilst the reverse inclusion is clear. 
We apply the lemma with $\left\{i_{1}, \ldots, i_{k}\right\}=\{1, \ldots n\}$, which tells us that $N_{1} \cap \ldots \cap N_{n}=$ $\prod_{i=1}^{n} R_{i} \cap N_{i}$, and so assuming independence of the $R_{i}$ we have

$$
N_{1} \cap \ldots \cap N_{n}=\prod_{i=1}^{n}\left[R_{i}, N_{i}\right] \subseteq[R, R] .
$$

$(2) \Rightarrow(1)$. There exist minimal and pairwise disjoint generating sets $\mathbf{r}_{i}$ for $R_{i}^{a b}$ as a $\mathbb{Z} G$-module, $(i=1, \ldots n)$, with preimages $\hat{\mathbf{r}}_{i} \subseteq F$ such that no two elements of $\hat{\mathbf{r}}_{1} \cup \ldots \cup \hat{\mathbf{r}}_{n}$ are $F$-conjugate. For each $i$ with $1 \leq i \leq n$ there is a short exact sequence

$$
0 \rightarrow \pi_{2} Y_{i} \rightarrow \bigoplus_{\mathbf{r}_{i}} \mathbb{Z} G_{i} \rightarrow R_{i}^{a b} \rightarrow 0
$$

where $Y_{i}$ is the standard 2-complex model of the presentation $\left\langle\mathbf{x} \mid \hat{\mathbf{r}}_{i}\right\rangle$ of $G_{i}$, and a short exact sequence

$$
0 \rightarrow \pi_{2} Y \rightarrow \bigoplus_{\mathbf{r}} \mathbb{Z} G \rightarrow R^{a b} \rightarrow 0
$$

where $\mathbf{r}=\mathbf{r}_{1} \cup \ldots \cup \mathbf{r}_{n}$ and $Y$ is the 2-complex model of the presentation $\langle\mathbf{x} \mid \hat{\mathbf{r}}\rangle$ of $G$. Tensoring each sequence (4) with $\mathbb{Z} G$ and summing over $i$ yields the commutative diagram

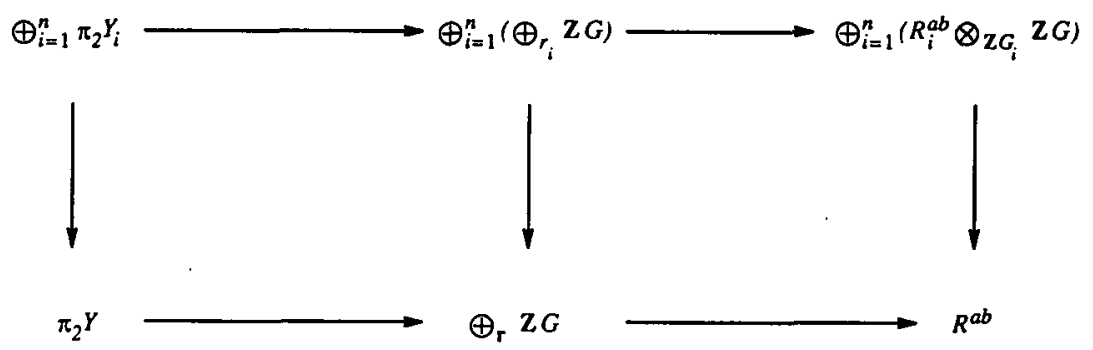

Figure 2.1

from which it follows that the map $\bigoplus_{i=1}^{n} \pi_{2} Y_{i} \stackrel{a}{\rightarrow} \pi_{2} Y$ is surjective.

Let $Z_{i}$ be the 2-complex model of the presentation

$$
\left\langle\mathbf{x} \mid \hat{\mathbf{r}}_{1} \cup \ldots \cup \hat{\mathbf{r}}_{i-1} \cup \hat{\mathbf{r}}_{i+1} \cup \ldots \cup \hat{\mathbf{r}}_{n}\right\rangle
$$

of $F / N_{i}$. Then the map $\alpha$ factors through $\pi_{2} Y_{i} \oplus \pi_{2} Z_{i} \stackrel{\beta}{\rightarrow} \pi_{2} Y$ which is therefore surjective: however, by [11] its cokernel is $R_{i} \cap N_{i} /\left[R_{i}, N_{i}\right]$.

In $[4,7]$ hyper-relative homology groups are considered for any group $F$ relative to a family of normal subgroups $\left\{N_{1}, \ldots, N_{n}\right\}$. We recall from [7] that the first hyper-relative homology group $H_{1}\left(F ; N_{1}, \ldots, N_{n}\right)$ may be defined algebraically as

$$
H_{1}\left(F ; N_{1}, \ldots, N_{n}\right)=\frac{N_{1} \cap \ldots \cap N_{n}}{\prod_{I \subseteq\{1, \ldots, n\}}\left[\bigcap_{i \in I} N_{i}, \bigcap_{j \notin I} N_{j}\right]},
$$

with $\bigcap_{\varnothing} N_{i}$ interpreted as $R$. 
Proposition 2.3. Let $F$ be a free group and $\left\{R_{1}, \ldots, R_{n}\right\}$ a family of independent normal subgroups of $F$, with $n>1$. Then

(a) $H_{1}\left(F ; N_{1}, \ldots, N_{n}\right)=0$,

(b) for each $i$ with $1 \leq i \leq n$, we have $H_{2}\left(R / N_{i}\right)=0=H_{2}\left(R / R_{i}\right)$.

Proof. (a) Recall from the proof of Theorem 2.1 that, assuming the independence of the $R_{i}$, we have $N_{1} \cap \ldots \cap N_{n}=\prod_{i=1}^{n}\left[R_{i}, N_{i}\right]$. We shall show that

$$
\prod_{i=1}^{n}\left[R_{i}, N_{i}\right] \subseteq \prod_{I \subseteq\{1, \ldots, n\}}\left[\bigcap_{i \in I} N_{i}, \bigcap_{j \notin l} N_{j}\right] .
$$

Consider a term in the product on the right-hand side with $I=\{i\}$. Then by Lemma 2.2 ,

$$
\left[N_{i}, \bigcap_{j \neq i} N_{j}\right]=\left[N_{i}, \prod_{j \neq i}\left(R_{j} \cap N_{j}\right) R_{i}\right]
$$

and the right-hand side here clearly contains $\left[N_{i}, R_{i}\right]$.

(b) We use Hopf's formula for $H_{2}$ : for $R / N_{i}$ this gives

$$
\begin{aligned}
H_{2}\left(R / N_{i}\right) & =H_{2}\left(R_{i} N_{i} / N_{i}\right) \\
& =\left(N_{i} \cap\left[R_{i} N_{i}, R_{i} N_{i}\right]\right) /\left[N_{i}, R_{i} N_{i}\right] \\
& =\left(N_{i} \cap\left[R_{i}, R_{i}\right]\left[N_{i}, N_{i}\right]\left[R_{i}, N_{i}\right]\right) /\left[N_{i}, N_{i}\right]\left[N_{i}, R_{i}\right] .
\end{aligned}
$$

But $\left[N_{i}, N_{i}\right]\left[N_{i}, R_{i}\right]=\left[N_{i}, N_{i}\right]\left(R_{i} \cap N_{i}\right)=N_{i} \cap\left[N_{i}, N_{i}\right] R_{i}$ which clearly contains $N_{i} \cap$ $\left[R_{i}, R_{i}\right]\left[N_{i}, N_{i}\right]\left[R_{i}, N_{i}\right]$. The proof that $H_{2}\left(R / R_{i}\right)=0$ proceeds in the same way.

\subsection{Examples}

\subsubsection{Free products}

Consider a free product $G=A_{1} * \ldots * A_{n}$. Given presentations $\left\langle\mathbf{x}_{i} \mid \mathbf{r}_{i}\right\rangle$ of the $A_{i}$, we obtain a presentation $\langle\mathbf{x} \mid \mathbf{r}\rangle$ of $G$, where $\mathbf{x}=\mathbf{x}_{1} \sqcup \ldots \sqcup \mathbf{x}_{n}$ and $\mathbf{r}=\mathbf{r}_{1} \sqcup \ldots \sqcup \mathbf{r}_{n}$. Let $F_{i}$ be the free group with basis $\mathbf{x}_{i}$ and $F$ the free group with basis $\mathbf{x}$ : then $R_{i}$ is the normal closure of $\mathbf{r}_{i}$ in $F$. We write $\Phi_{i}$ for the free group $*_{j \neq i} F_{j}$. Let $M_{i}$ be the relation module of the presentation $\left\langle\mathbf{x}_{i} \mid \mathbf{r}_{i}\right\rangle$, that is, the $A_{i}$-module $\left.\left(\left\langle\mathbf{r}_{i}\right\rangle\right\rangle^{F_{i}}\right)^{a b}$ : then the following three equivalent statements hold.

(a) the normal subgroups $R_{1}, \ldots, R_{n}$ are independent,

(b) the inclusions $\mathbf{r}_{i} \leftrightarrow \mathbf{r}$ induce an isomorphism

$$
R^{a b} \cong \bigoplus_{i=1}^{n} M_{i} \otimes_{A_{i}} \mathbb{Z} G
$$

of $\mathbb{Z} G$-modules,

(c) the inclusions $\mathbf{r}_{i} \leftrightarrow \mathbf{r}$ induce an isomorphism

$$
\left.R^{a b} \cong \bigoplus_{i=1}^{n}\left(\left\langle\mathbf{r}_{i}\right\rangle\right)^{F}\right)^{a b} \otimes_{G_{i}} \mathbb{Z} G
$$

of $\mathbb{Z} G$-modules, where $G_{i}$ is the free product $\Phi_{i} * A_{i}$ with presentation $\left\langle\mathbf{x} \mid \mathbf{r}_{i}\right\rangle$. 
The equivalence of (b) and (c) follows from the group isomorphism $R_{i} \cong$ $*_{t \in T} t^{-1}\left\langle\left\langle\mathbf{r}_{i}\right\rangle\right)^{F_{i}}$, where $T$ is a transversal for the double cosets $\left.F_{i} \backslash F /\left\langle\mathbf{r}_{i}\right\rangle\right\rangle^{F_{i}}$, and the equivalence of (a) and (c) follows from Theorem 2.1. It is easy to deduce (b) from the relation sequence

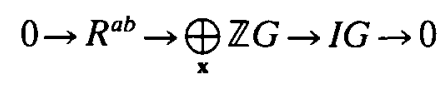

and the decomposition of the augmentation ideal $I G$ as $I G \cong \bigoplus_{i=1}^{n} I A_{i} \otimes_{A_{i}} \mathbb{Z} G$, see [12].

\subsubsection{Efficient factorizations of free groups}

Let $\langle\mathbf{x} \mid \mathbf{r}\rangle$ be a balanced finite presentation of the trivial group, and let $F$ be the free group with basis $\mathbf{x}$. For any partition $\mathbf{r}=\mathbf{r}_{1} \sqcup \ldots \sqcup \mathbf{r}_{n}$, we let $R_{i}$ denote the normal closure in $F$ of $\mathbf{r}_{i}$. Bogley [1] calls the product $F=R_{1} \ldots R_{n}$ an efficient factorization of $F$ and conjectures that if $R$ and $S$ are distinct factors in an efficient factorization then $R \cap S=[R, S]$. Bogley proves this conjecture for $n=2$, and this case shows that the factors in an efficient factorization of a finitely generated free group are independent. Here we have a good source of examples. If Bogley's conjecture is true, then the factors of an efficient factorization are an independent set of normal subgroups every subset of which is also independent.

3. Independence and one-relator products. A one-relator product is the quotient of a free product by the normal closure of a single element. Let $G=A * B /\langle w\rangle$ be a one-relator product, where $w$ is cyclically reduced and of length at least 2 in $A * B$. We write $w=u^{m}$ for $m \geq 1$ as large as possible, so that $u$ is not a proper power in $A * B$. Howie $[13,14,15]$ and Duncan and Howie [6] have established relation module decompositions for certain one-relator products. The group $G$ is said to be exceptional of type $E(p, q, m)$ if some cyclic permutation of $u \in A * B$ can be written as $a V b V^{-1}$ with $a, b \in A \sqcup B$ and $V \in A * B$ such that $a^{p}=b^{q}=1$ in $A * B$ and $1 / p+1 / q+1 / m>1$. Note that if $G$ is exceptional then there are at least two cyclic permutations of $u$ of the given form, namely $a V b V^{-1}$ and $b V^{-1} a V$. If there are exactly two such cyclic permutations of $u$ then $G$ is uniquely exceptional.

We obtain a presentation for $G$, from given presentations $\left\langle\mathbf{x}_{1} \mid \mathbf{r}_{1}\right\rangle$ and $\left\langle\mathbf{x}_{2} \mid \mathbf{r}_{2}\right\rangle$ for $A$ and $B$ respectively, in the following way. Let $F$ be the free group with basis $\mathbf{x}=\mathbf{x}_{1} \sqcup \mathbf{x}_{2}$. We replace $u$ by a word in $F$ mapping to it, and so obtain the presentation $\left\langle\mathbf{x} \mid \mathbf{r}_{1}, \mathbf{r}_{2}, u^{m}\right\rangle$ of $G$. We let $F_{i}$ be the free group with basis $\mathbf{x}_{i}$ and set $G_{1}=A * F_{2}, G_{2}=F_{1} * B$. We set $\left.R_{i}=\left\langle\mathbf{r}_{i}\right\rangle\right\rangle^{F}, R_{3}=\left\langle\left\langle u^{m}\right\rangle\right\rangle^{F}$ and $R=\left\langle\left\langle\mathbf{r}_{1}, \mathbf{r}_{2}, u^{m}\right\rangle\right\rangle^{F}$.

Suppose that either

(1) $m \geq 4$ and that $G$ is not exceptional [14], or

(2) that $m \geq 3$ and $u \in A * B$ contains no letter of order 2 [6], or

(3) $A$ and $B$ are locally indicable [13].

Then the relation module $R^{a b}$ decomposes as a $G$-module as

$$
\left(M_{A} \otimes_{A} \mathbb{Z} G\right) \oplus\left(M_{B} \otimes_{B} \mathbb{Z} G\right) \oplus \mathbb{Z} G /(1-u)
$$


where $M_{A}=\left(\left\langle\left\langle\mathbf{r}_{1}\right\rangle\right\rangle^{F_{1}}\right)^{a b}$ and $M_{B}=\left(\left\langle\left\langle\mathbf{r}_{2}\right\rangle\right\rangle^{F_{2}}\right)^{a b}$. It follows that $R^{a b}$ is isomorphic to

$$
\left(R_{1}^{a b} \otimes_{G_{1}} \mathbb{Z} G\right) \oplus\left(R_{2}^{a b} \otimes_{G_{2}} \mathbb{Z} G\right) \oplus\left(R_{3}^{a b} \otimes_{G_{3}} \mathbb{Z} G\right)
$$

where $G_{3}$ is the one-relator group $\left\langle\mathbf{x} \mid u^{m}\right\rangle$, and by Lyndon's Identity Theorem, $R_{3}^{a b} \cong \mathbb{Z} G_{3} /(1-u)$. From Theorem 2.1 we deduce the following result.

THEOREM 3.1. Suppose that one of the three conditions (1) to (3) above holds. Then the family $\left\{R_{1}, R_{2}, R_{3}\right\}$ is independent.

If $u \notin[F, F]$ then $H_{i}(F / N)=H_{i}(A) \oplus H_{i}(B),(i=1,2,3,4), H_{2}(F / M)=0=H_{4}(F / M)$ and $H_{3}(F / M)=\mathbb{Z}_{m}$. It then follows from the calculations of $H_{i}(G)$ in $[6,14,15]$ that in Theorem 1.1 (in which we set $\Gamma=F, M=R_{3}$ and $N=R_{1} R_{2}$ ) the terms $V_{2}$ and $V_{3}$ are zero. If $u \in[F, F]$ then $H_{2}(F / M)=\mathbb{Z}$. However, as pointed out to us by Bogley, it is still true that $V_{2}=0=V_{3}$. For the calculations of second homotopy groups in $[6,14,15]$ imply that the map

$$
H_{2}(A) \oplus H_{2}(B) \oplus H_{2}(F / M) \rightarrow H_{2}(G)
$$

is injective and that for $i>2$ we have $H_{i}(F / M)=H_{i}\left(\mathbb{Z}_{m}\right)$. From these facts it follows that $V_{2}=0=V_{3}$.

In the case where $G$ is a uniquely exceptional group and $m \geq 4$ it is still possible to determine the structure of $R_{3} \cap N_{3} /\left[R_{3}, N_{3}\right]$, although $\left\{R_{1}, R_{2}, R_{3}\right\}$ is not independent. Recall from [11] that $R_{3} \cap N_{3} /\left[R_{3}, N_{3}\right] \cong \operatorname{coker}(\beta)$, where for suitably chosen complexes $Y_{3}, Z_{3}$ and $Y$, we have $\beta: \pi_{2} Y_{3} \otimes \pi_{2} Z_{3} \rightarrow \pi_{2} Y$. In fact, as before, we can take $Y_{3}, Z_{3}$ and $Y$ to be the standard 2-complex models of $\left\langle\mathbf{x} \mid u^{m}\right\rangle$ and $\left\langle\mathbf{x} \mid \mathbf{r}_{1}, \mathbf{r}_{2}\right\rangle$ and $\left\langle\mathbf{x} \mid \mathbf{r}_{1}, \mathbf{r}_{2}, u^{m}\right\rangle$, respectively. If $G$ is uniquely exceptional with $m \geq 4$, then it follows from Section 4 of [14] that $R_{3} \cap N_{3} /\left[R_{3}, N_{3}\right]$ is generated as a $G$-module by a single element.

The detailed argument involves the representation of elements of $\pi_{2} Y$ by spherical pictures over $G$, and the generator for $R_{3} \cap N_{3} /\left[R_{3}, N_{3}\right]$ is obtained from a picture of type $\Gamma(p, q, m)$ over $G$, see [14]. In terms of pictures, the quotient map $\pi_{2} Y \rightarrow R_{3} \cap$ $N_{3} /\left[R_{3}, N_{3}\right]$ amounts to choosing an equator [2] on a spherical picture $\Gamma$. To construct an equator we choose a minimal subtree $T$ of $\Gamma$ (regarding vertices as 0 -dimensional) and then choose a regular neighbourhood $N$ of $T \cup$ vertices of $\Gamma$ such that no region of $\Gamma$ is contained in $N$. The boundary of the closure of $N$ is then an equator on $\Gamma$. The equator bounds a picture over $G$ on the disc, the boundary label of which is an element of $R_{3} \cap N_{3}$.

The word labelling the boundary can be of considerable complexity, which we illustrate with $G$ uniquely exceptional of type $E(2,3,4)$. Here $u=a V b V^{-1}$ with $a^{2}=b^{3}=1$. Let $c=V^{-1} a V$ : then $\left(R_{3} \cap N_{3}\right) /\left[R_{3}, N_{3}\right]$ is generated over $G$ by

$$
c\left((b c)^{3} b\right)\left((b c)^{2} b\right) b\left((b c)^{2} b\right) b(b c)^{4}\left(c(b c)^{3}\right)(c(b c))\left(c(b c)^{3}\right)\left(c(b c)^{3}\right)
$$

This is the label on an equator of the $\Gamma(2,3,4)$ picture shown in Figure 3.1. 


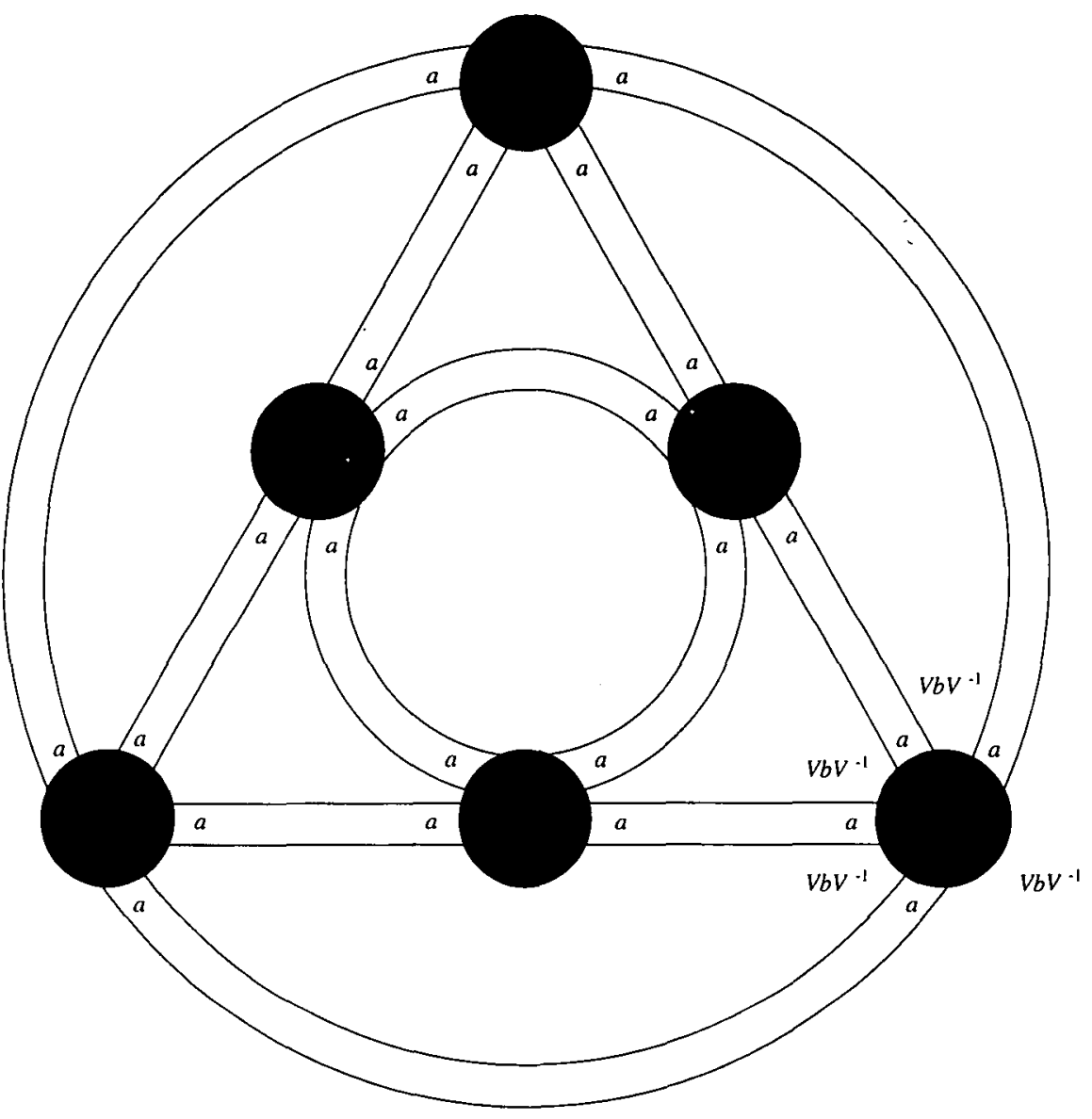

Figure 3.1

\section{REFERENCES}

1. W. A. Bogley, An embedding for $\pi_{2}$ of a subcomplex of a finite contractible two-complex. Glasgow Math. J. 33 (1991), 365-372.

2. W. A. Bogley and M. A. Gutierrez, Mayer-Vietoris sequences in homotopy of 2-complexes and in homology of groups. J. Pure Appl. Algebra 77 (1992), 39-65.

3. W. A. Bogley and S. J. Pride, Calculating generators of $\pi_{2}$, in Two-dimensional Homotopy and Combinatorial Group Theory, C. Hog-Angeloni et al. (Eds.). London Math. Soc. Lecture Notes 197, Cambridge University Press (1993).

4. R. Brown and G. J. Ellis, Hopf formulae for the higher homology of a group. Bull. London Math. Soc. 20 (1988) 124-128.

5. R. Brown and J.-L. Loday, Van Kampen Theorems for diagrams of spaces. Topology 26 (1987) 311-335.

6. A. J. Duncan and J. Howie, Weinbaum's conjecture on unique subwords of non-periodic words. Proc. Amer. Math. Soc. 115 (1992) 947-954.

7. G. J. Ellis, Relative derived functors and the homology of groups. Cahiers Top. Geom. Diff. 31(2) (1990) 121-135. 
8. G. J. Ellis and R. Steiner, Higher-dimensional crossed modules and the homotopy groups of $(n+1)$-ads. J. Pure Appl. Algebra 46 (1987) 117-136. $263-276$.

9. N. D. Gilbert, Identities between sets of relations. J. Pure Appl. Algebra 83 (1993)

10. K. W. Gruenberg, Relation Modules of Finite Groups. CBMS Monograph 25 (American Mathematical Society, Providence RI, 1976).

11. M. A. Gutierrez and J. G. Ratcliffe, On the second homotopy group. Quart. J. Math. Oxford (2) 32 (1981) 45-55.

12. P. J. Hilton and U. Stammbach, A Course in Homological Algebra, (Springer-Verlag, Berlin-Heidelberg-New York 1971).

13. J. Howie, Cohomology of one-relator products of locally indicable groups. J. London Math. Soc. (2) 30 (1984) 419-430.

14. J. Howie, The quotient of a free product of groups by a single high-powered relator. I. Pictures. Fifth and higher powers. Proc. London Math. Soc. (3) 59 (1989) 507-540. Corrigendum. Proc. London Math. Soc. (3) 66 (1993) 538.

15. J. Howie, The quotient of a free product of groups by a single high-powered relator. II. Fourth powers. Proc. London Math. Soc. (3) 61 (1990) 33-62.

16. J. Huebschmann, Aspherical 2-complexes and an unsettled problem of J. H. C. Whitehead. Math. Ann. 258 (1981) 17-37.

17. P. A. Linnell, Decomposition of augmentation ideals and relation modules. Proc. London Math. Soc. (3) 47 (1983) 83-127.

18. C. Miller, The second homology of a group: relations between commutators. Proc. Amer. Math. Soc. 3 (1952) 588-595.

19. J. H. C. Whitehead, A certain exact sequence. Ann. of Math. 52 (1950) 51-110.

A. J. DUNCAN

Department of Mathematics \& Statistics

UNIVERSITY of NEWCASTLE UPON TYNE

NEWCASTLE UPON TYNE NE1 7RU

ENGLAND

N. D. GILbERT

Department of Mathematical Sciences

DURHAM UNIVERSITY

Science Laboratories, South Road

DURHAM DH1 3LE

ENGLAND
Graham J. Ellis Department of Mathematics UNIVERSITY COLLEGE Galway IRELAND 